\title{
Pressed ceramic in fixed partial dentures construction
}

\author{
Marginal adaptation of three-unit fixed partial dentures constructed from pressed ceramic systems \\ C. F. J. Stappert, M. Dai, S. Chitmongkolsuk, T. Gerds and J. R. Strub Br Dent J 2004; 196: 766-770
}

\section{Purpose}

This study compares the marginal accuracy of posterior metal ceramic (MC), all-ceramic IPS Empress ${ }^{\circledR} 2$ and experimental pressed ceramic (EPC-VP 1989/4) three-unit fixed partial dentures (FPD), before and after luting and after thermo-mechanical fatigue in a dual-axis chewing simulator.

\section{Materials and methods}

Caries-free human teeth $(n=160)$ were used as abutments for the fabrication of eighty posterior three-unit FPD, divided into two test-groups, IPS Empress ${ }^{\circledR} 2$ and EPC, of 32 samples each and one control group of 16 samples metal ceramic FPD. All FPD were cemented with Variolink ${ }^{\circledR}$ II dual-curing resin cement. Half of the samples in each group were exposed to a dual-axis chewing simulator.

\section{Results}

The geometric mean marginal gap values ( $\mu \mathrm{m}$, before cementation, after cementation and after thermo-mechanical fatigue) amounted to 53, 63 and 62 for the ceramic metal FPD, 57, 71 and 68 for the Empress ${ }^{\circledR} 2$ FPD and 55, 67 and 68 for the EPC FPD. In all groups a statistically significant increase in marginal gap width was observed after cementation. The effect of functional loading in the chewing simulator on marginal gap was not significant. Marginal gap was lowest in the control group but differences with all-ceramic materials were small in all evaluation stages.

\section{Conclusion}

Within the limits of this investigation, it can be concluded that marginal gap values of these all-ceramic materials and conventional MC techniques are on a similar level. In particular, almost all marginal gap values observed in this study were within the limits of clinical acceptance.

\section{IN BRIEF}

- Within the limits of this investigation, it can be concluded that marginal accuracy of pressed all-ceramic FPD and the conventional $M C$ techniques used are on a similar level.

- In particular, almost all marginal gap values observed in this study were in the range of 50-100 $\mu \mathrm{m}$, which is ideal for composite cement and seems to optimise performance.

- The process of cementation reduces the marginal accuracy of FPD restorations irrespective of the FPD material.

- After artificial aging marginal degradation of cement films was not significant using a resin-cement and dentine-bonding system for cementing the all-ceramic FPD. Therefore, the investigators judged resin-cements as the most suitable for cementing all-ceramic restorations.

\section{COMMENT}

Ceramics have developed enormously in recent years and when combined with new processing techniques this has created new opportunities for the dental application of ceramics. Consequently, the all-ceramic fixed partial denture (FPD) is gaining popularity as an alternative to the well established metal-ceramic bridge. One approach involves using a high strength ceramic rather than metal core, which is glazed with an aesthetic veneer and then cemented in place using conventional luting cements. Versions of these are the glass infiltrated alumina or zirconia cores and core systems made of pure alumina or yttria stabilised zirconia. The system explored in the paper by Stappert et al. is different in that it is based around a resin-bonded ceramic core, not a cemented core, and is made of a lithium disilicate glass-ceramic produced by a hot pressing route.

At present there is little clinical information that can guide the practitioner in the decision making process about new all-ceramic FPD systems. The evidence base consists primarily of laboratory data of fundamental materials properties such as strength and toughness or, as is the case with this paper, data derived from a simulation of the clinical situation. The authors chose to compare the performance of two resin-bonded ceramic three unit FPDs and a cemented metal-ceramic bridge. They assessed how such bridges would perform under simulated cyclic loading conditions using the marginal integrity as the assessment parameter. The marginal integrity was assessed by measurement of the marginal gap and it was found that immediately before and after cementation there was no significant difference in the marginal gap, in the region of 50-70 $\mathrm{mm}$ for all three systems and considered to be well within clinically acceptable limits. Thus the quality of construction of the FDPs with respect to their marginal fit is very acceptable.

Fatigue failure due to cyclic or thermal loading is now recognised as a potentially significant contributor to the ultimate failure of dental restorations and this paper shows that thermomechanical cyclic loading does not affect the marginal accuracy as determined by no change in the marginal gap. This is very reassuring. However, it should not be assumed from this observation that the restorations are unaffected by the simulated loading conditions as marginal accuracy is only one aspect of many factors contributing to the clinical performance.

For the resin-bonded all-ceramic FPDs an important consideration is the integrity of the bond between the resin and the ceramic. If this is lost then its structural strength can be seriously compromised. Examination of the marginal accuracy will not necessarily pick this up and thus we must look forward to more work being done in this area so as to reassure us that allceramic FPDs will deliver a clinical performance that will be acceptable. This paper represents an important step in the right direction.

R. Van Noort, Adult Dental Care,

University of Sheffield School of Clinical Dentistry

doi:10.1038/sj.bdj.4811388 